\title{
RESEARCH HIGHLIGHT OPEN Targeting NR4As, a new strategy to fine-tune CAR-T cells against solid tumors
}

\author{
Feng $\mathrm{Li}^{1}$ and Yi Zhang ${ }^{1}$ \\ Signal Transduction and Targeted Therapy (2019)4:7; https://doi.org/10.1038/s41392-019-0041-1
}

In a recent report in Nature, Chen et al. ${ }^{1}$ identified the critical transcription factors that drive T-cell dysfunction. Knockout of these transcription factors to augment T-cell activity represents a new approach to enhancing the effectiveness of chimeric antigen receptor T (CAR-T) cell therapy for solid tumors.

CAR-T cells are designer cytotoxic lymphocytes with recognition specificity and tumoricidal potency. Although CAR-T cells exhibit unprecedented effectiveness in treating hematologic malignancies, these cells have limited efficacy against solid tumors. ${ }^{2}$ Therefore, intensive efforts have been made to augment the efficiency of CAR-T cell therapy for solid tumors.

A major barrier in solid tumors is the hostile microenvironment surrounding CAR-T cells. To overcome the immunosuppressive effects within the tumor niche, several tactics have been undertaken. The administration of programmed cell death protein 1 (PD-1) antibodies is effective in restoring CAR-T cell function. ${ }^{3}$ Recently, CAR-T cells armed with a secreted minor fragment derived from the variable domains of a PD-1-blocking antibody were tested. ${ }^{4}$ This design seemed more efficient to augment the functionality of host and nearby $T$ cells than did the full-length antagonist. Soluble transforming growth factor- $\beta$ (TGF- $\beta$ ) is also important for inhibiting T-cell activation. By introducing signal-switch receptors comprising the TGF- $\beta$-binding ectodomain and the T-cell activation endodomain, CAR-T cells are more potently activated in the tumor microenvironment. ${ }^{5}$ The suppressive immune cells represent another obstacle for CAR-T cell therapy. With enforced expression of interleukin (IL)-18, CAR$T$ cells are more active against tumors by decreasing the populations of suppressive regulatory $T\left(T_{\text {reg }}\right)$ cells and $M 2$ macrophages. $^{6}$

T-cell differentiation is also the determinant for therapeutic efficacy. Less differentiated T cells have better persistence in vivo. Nevertheless, a large portion of T cells are terminally differentiated during CAR-T cell production. To augment the populations of more proliferative T cells with stem-cell-like or memory phenotypes, specific supplements were added in culture. The addition of IL-7 and IL-15 but not IL-2 induces the generation of more memory cells without compromising the cytotoxic function. ${ }^{7}$ Similarly, supplementation with a phosphoinositide 3-kinase- $\delta$
(PI3k- $\delta$ ) antagonist shows promise in preserving memory subsets during CAR-T cell expansion. ${ }^{8}$ Alternatively, an infusion with naive $\mathrm{T}$ cells, which subsequently differentiate in vivo, may result in better clinical responses. The combination of CAR-modified induced pluripotent stem cells (iPSCs) and organoid-dictated Tcell lineage commitment makes this hypothesis possible. A previous group established such an approach to produce naive CAR-T cells, ${ }^{9}$ which may introduce new scenarios in treating tumors.

Recently, a report published in Nature proposed a new strategy to improve CAR-T cell therapy. ${ }^{1}$ To avoid autoimmunity, various transcription factors are upregulated to limit the intensity and interval of T-cell activation. Such factors will upregulate the expression of inhibitory receptors, subsequently induce terminal differentiation and downregulate the secretion of inflammatory cytokines. Hence, finding the key intrinsic initiator of T-cell dysfunction can provide new targets for the preparation of upgraded CAR-T cells. Chen et al. ${ }^{1}$ uncovered NR4A1, NR4A2 and NR4A3 as the central transcriptional factors that drive T-cell dysfunction. To identify the intrinsic triggers of exhaustion, these authors checked the transcriptional adaptations between functional and hypofunctional $\mathrm{CD}^{+} \mathrm{T}$ cells. By analyzing single cell transcriptome and chromatin accessibility, the researchers discovered that the transcription factors NR4A1, NR4A2 and NR4A3 were upregulated and played critical roles in mediating $\mathrm{CD}^{+}{ }^{+}$-cell exhaustion in mouse CAR-modified and endogenous lymphocytes and their human counterparts exposed to tumor or chronic virus infection. When all three NR4As were deficient, the activated $\mathrm{CD}^{+} \mathrm{T}$ cells demonstrated features resembling effector T cells with increased functionality rather than those with an exhausted status. Additionally, the researchers generated CAR-T cells with a deletion of NR4As. Compared with their wild-type counterparts, the NR4A-deficient CAR$T$ cells showed enhanced cytotoxicity but decreased inhibitory receptor expression and promoted tumor regression more significantly.

This finding provides us with new candidate targets to augment the cytotoxic capacities of CAR-T cells during chronic antigen engagement, which supplements the approaches that help to improve the effectiveness of adoptive transfer therapy for solid tumors (Fig. 1).

\footnotetext{
${ }^{1}$ Biotherapy Center and Cancer Center, The First Affiliated Hospital of Zhengzhou University, Zhengzhou, Henan, China Correspondence: Yi Zhang (yizhang@zzu.edu.cn)
}

Received: 9 March 2019 Accepted: 12 March 2019

Published online: 29 March 2019 


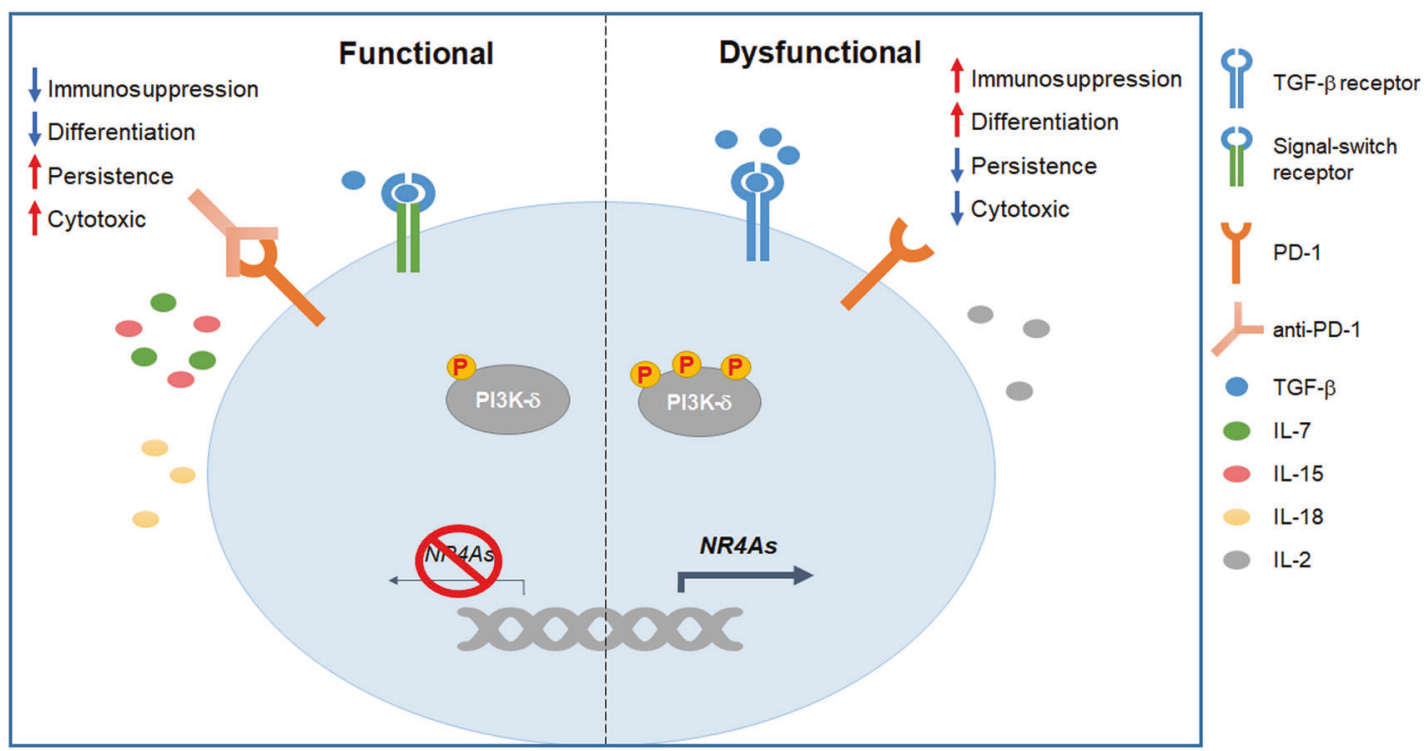

Fig. 1 Strategies to augment chimeric antigen receptor T (CAR-T) cell activity in treating solid tumors. Dysfunction of CAR-T cells is related to many intrinsic and extrinsic factors (right). Maintenance of the proliferative $T$ cells and reversion of the inhibition signals are reasonably needed to sustain CAR-T cell function within the tumor (left). Manipulation of the genes that exacerbate T-cell exhaustion is also effective in improving the efficacy of CAR-T cell therapy (left)

\section{REFERENCES}

1. Chen, J. et al. NR4A transcription factors limit CAR T cell function in solid tumours. Nature https://doi.org/10.1038/s41586-019-0985-x (2019).

2. June, C. H. \& Sadelain, M. Chimeric antigen receptor therapy. N. Engl. J. Med. 379, 64-73 (2018)

3. Cherkassky, L. et al. Human CAR T cells with cell-intrinsic PD-1 checkpoint blockade resist tumor-mediated inhibition. J. Clin. Invest. 126, 3130-3144 (2016).

4. Rafiq, S. et al. Targeted delivery of a PD-1-blocking ScFv by CAR-T cells enhances anti-tumor efficacy in vivo. Nat. Biotechnol. 36, 847-856 (2018).

5. Sukumaran, S. et al. Enhancing the potency and specificity of engineered T cells for cancer treatment. Cancer Discov. 8, 972-987 (2018).

6. Chmielewski, M. \& Abken, H. CAR T cells releasing IL-18 convert to T-Bet(high) FoxO1(low) effectors that exhibit augmented activity against advanced solid tumors. Cell Rep. 21, 3205-3219 (2017).

7. Xu, Y. et al. Closely related T-memory stem cells correlate with in vivo expansion of CAR.CD19-T cells and are preserved by IL-7 and IL-15. Blood 123, 3750-3759 (2014).

8. Abu Eid, R. et al. Enhanced therapeutic efficacy and memory of tumor-specific CD8 T cells by ex vivo PI3K-delta inhibition. Cancer Res. 77, 4135-4145 (2017).
9. Montel-Hagen, A. et al. Organoid-induced differentiation of conventional T cells from human pluripotent stem cells. Cell Stem Cell 24, 376-389.e8 (2019).

(c) (P)

Open Access This article is licensed under a Creative Commons Attribution 4.0 International License, which permits use, sharing, adaptation, distribution and reproduction in any medium or format, as long as you give appropriate credit to the original author(s) and the source, provide a link to the Creative Commons license, and indicate if changes were made. The images or other third party material in this article are included in the article's Creative Commons license, unless indicated otherwise in a credit line to the material. If material is not included in the article's Creative Commons license and your intended use is not permitted by statutory regulation or exceeds the permitted use, you will need to obtain permission directly from the copyright holder. To view a copy of this license, visit http://creativecommons. org/licenses/by/4.0/.

(c) The Author(s) 2019 